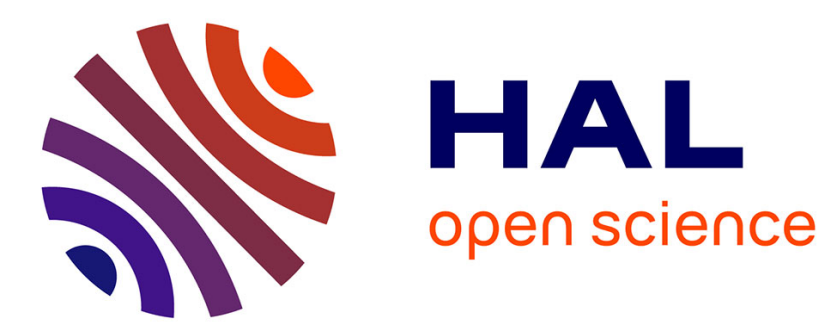

\title{
Retinoid X receptors: common heterodimerization partners with distinct functions.
}

\author{
Philippe Lefebvre, Yacir Benomar, Bart Staels
}

\section{To cite this version:}

Philippe Lefebvre, Yacir Benomar, Bart Staels. Retinoid X receptors: common heterodimerization partners with distinct functions.. Trends Endocrinol Metab, 2010, 21 (11), pp.676-83. 10.1016/j.tem.2010.06.009 . inserm-00521590

\section{HAL Id: inserm-00521590 https://www.hal.inserm.fr/inserm-00521590}

Submitted on 28 Sep 2010

HAL is a multi-disciplinary open access archive for the deposit and dissemination of scientific research documents, whether they are published or not. The documents may come from teaching and research institutions in France or abroad, or from public or private research centers.
L'archive ouverte pluridisciplinaire HAL, est destinée au dépôt et à la diffusion de documents scientifiques de niveau recherche, publiés ou non, émanant des établissements d'enseignement et de recherche français ou étrangers, des laboratoires publics ou privés. 


\title{
Retinoid X Receptors: common
}

\section{heterodimerization partners with distinct functions.}

\author{
Philippe Lefebvre ${ }^{1,2,3,4}$, Yacir Benomar ${ }^{1,2,3,4,5}$, Bart Staels ${ }^{1,2,3,4}$ \\ ${ }^{1}$ Univ Lille Nord de France, F-59000, Lille, France; ${ }^{2}$ INSERM, UMR1011, F-59000, Lille, France; ${ }^{3}$ \\ UDSL, F-59000, Lille, France; ${ }^{4}$ Institut Pasteur de Lille, F-59019, Lille, France;
}

\footnotetext{
${ }^{5}$ Present address: Centre des Neurosciences Paris Sud, CNRS UMR 1197, Université Paris Sud-XI, F-91405 ORSAY, France
} 


\section{Abstract}

Nuclear receptor biology and pathophysiology studies have emphasized the roles of retinoid $X$ receptors (RXRs) in cellular processes ranging from cellular proliferation to lipid metabolism. These pleiotropic effects stem not only from RXR's ability to dimerize with various nuclear receptors, which exert transcriptional control on specific aspects of cellular biology, but also from the permissive nature of some RXR heterodimers, reflecting the ability of RXR ligands to activate this heterodimer. This signaling network is rendered more complex by $R X R$ isotypes ( $R X R \alpha, R X R \beta, R X R \gamma)$ which have some distinctive properties, thereby modulating RXR-containing heterodimer transcriptional activities. This review discusses the emerging roles of RXR isotypes in the RXR signaling network, with possible implications in our understanding of nuclear receptor biology and pharmacology.

\section{Overview of RXR features}

The retinoid $X$ receptors (RXRs) are encoded by three distinct genes located on human chromosomes 9 (RXRA, NR2B1), 6 (RXRB, NR2B2) and 1 (RXRG, NR2B3), or on mouse chromosomes 2, 17 and 1 respectively, and share strong homologies in their coding sequences. RXRs have been initially characterized as heterodimeric partners of the retinoic acid (RARs), thyroid hormone (T3Rs) and vitamin D (VDR) nuclear receptors (NRs) and are required for high affinity binding to DNA of these and other RXR heterodimers ${ }^{1}$. NRs heterodimerizing with RXRs define a subclass which can be activated, or not, through RXR agonism. This (non)permissivity of RXR heterodimers underlines the central and complex roles of RXR in multiple biological processes and also the difficulty for precisely assessing their importance. The realization that RXRs could form homotetramers in solution and bind to DNA as homotetramers and homodimers ${ }^{2,3}$ led to the speculation that RXRs may control their own specific signaling pathways, rendering the analysis of the RXR pathway even more complex. Initial genetic analysis of RXR functions essentially pointed to their roles in embryonic development, which is believed to be conveyed mostly through RXR-RARs dimers ${ }^{4}$, and driven by the activation of RARs by the morphogen molecule all trans retinoic acid (atRA) ${ }^{5}$. Whereas the biological relevance and functions of atRA are nowadays undisputed, the 9-cis retinoic acid isomer, which was initially identified as a bona fide RXR ligand in vitro ${ }^{1}$, has never been 
detected in vivo ${ }^{6}$. This prompted the search for novel natural ligands, among which the unsaturated fatty acid docosahexanoic acid $(\mathrm{DHA})^{7}$ and 80 -apo14'-carotenal ${ }^{8}$ activate or repress $\mathrm{RXR}$ transcriptional activity respectively. However, these natural molecules are not RXR-specific ligands (rexinoids) and regulate notably the transcriptional activity of peroxisome proliferator-activated receptors (PPARs). The synthesis of rexinoids has revealed their potential as chemotherapeutic agents, exemplified by the successful use of Targretin ${ }^{\circledR}$ (bexarotene) in T-cell lymphoma treatment ${ }^{9}$. However, the promiscuous nature of RXRs has hindered the further development of rexinoids, especially in light of their propensity to cause hypertriglyceridemia ${ }^{10}$, a risk factor of cardiovascular diseases in spite of their beneficial effects on insulin sensitivity and fat mass.

As for all other NRs, the RXR ligand binding domain folds into a three-layered co-helical sandwich defining a hydrophobic ligand-binding pocket (LBP). In the unliganded state, the AF-2 AD (helix 12) is exposed to the solvent and yields free access of the ligand to the LBP (Figure 1). The RXR LBP is defined by the amino termini of helices 3 and 9 , the two beta sheets, helix 8 , the H8-H9 loop, and the carboxyl terminus of helix $11^{11}$. In analogy with RARs, the synthesis of RXR isotype-specific ligands would have been highly desirable. Unfortunately, the strong conservation of LBD and of LBP (ca. $500 \AA^{3}$ ) between RXR isotypes does not allow an easy design of isotype-selective ligands. However, the design of heterodimer-selective rexinoids ${ }^{12-14}$ demonstrated that activation of permissive heterodimers through $\mathrm{RXR}$ is a viable option for the pharmacological and possibly therapeutical control of dysregulated cellular pathways ${ }^{15}$. Furthermore, the control of RXR heterodimer non-permissivity through the activation of kinase signaling pathways (desubordination) provides another tool to modulate RXR heterodimers transcriptional activity ${ }^{16}$. In this review, we will discuss RXR biology and highlight recent advances in this field to forecast how this increased knowledge may create new options for drug discovery.

\section{RXR isotypes: similar but different.}

Tissue-specific distribution. The Rxra, Rxrb and Rxrg genes are differently expressed in mouse tissues: Rxra is the major isotype in mouse liver, whereas Rxrb expression is highest in the central nervous system. Rxrg displays a more restricted expression territory, with detectable levels in skeletal muscle and regions of the CNS such as the olfactory bulb and the pituitary glands ${ }^{17}$. 
RXRs are expressed as 3 isotypes and numerous isoforms resulting mostly from alternative splicing (Figure 1). The predominant mouse $R X R \alpha$ isoform is $R X R \alpha 1$, which encodes a $52 \mathrm{kDa}$ receptor. Two additional isoforms have been isolated from adult mouse testis, $R X R \alpha 2$ and RXR $\alpha 3$, which have a 28 and a 97 amino acid deletion in the N-terminal AF1 domain respectively ${ }^{18}$. Ensembl database inspection also identifies a fourth splice variant $\cos 4$ potentially generating a 165 aminoacid protein which has not been characterized functionally. Similarly, four mouse RXR $\beta$ isoforms have been identified. The human homolog of mRXR\&01, hRXR\&02, is conserved across species but contains two ATG initiator codons which may produce $\mathrm{N}$-terminal variants ${ }^{19}$. A third isoform, initially termed $\mathrm{hRXR} \beta 3$, contains a SLSR sequence inserted in the ligand-binding domain through the use of an alternative 3 '-splice acceptor site, similar to the mouse RXR $\beta 2 E$ isoform ${ }^{20}$. This insertion impacts negatively on the ligand binding activity of $R X R \beta$ and on its transactivation potential. Finally, $m R X R \gamma 1$ is strongly expressed in the brain and muscle, whereas the mRXR 2 isoform has a unique $5^{\prime}$-untranslated region and is expressed highly in both cardiac and skeletal muscles ${ }^{21}$.

The respective role(s) of RXR isotypes and isoforms is not fully understood. Germ line mutations in the Rxr genes induce either in utero lethality through congenital heart defects $(R X R \alpha)$, or metabolic and behavioral defects $\left[\mathrm{RXR} \beta, \mathrm{RXR} \gamma\right.$, reviewed in $\left.{ }^{22}\right]$. From a developmental point of view, $R X R \alpha$ seems to be the main player since mice expressing only $R X R \alpha$ from one allele are viable, indicating a strong functional redundancy ${ }^{23}$. Tissue-specific inactivation of $R x r a$ in hepatocytes ${ }^{24-28}$ or epithelia ${ }^{29,30}$ induces strong phenotypes, indicating a major role of RXR $\alpha$ in these tissues, whereas Rxra gene knockout has only a mild effect in hematopoietic progenitors ${ }^{31}$. The conditional ablation of $\mathrm{RXR} \alpha$ in adipocytes revealed that this isotype is necessary for adipocyte lipogenesis and hypertrophy, a phenomenon observed in obese animals, but not for adipocyte survival, for which $\mathrm{RXR} \gamma$ can compensate for RXR $\alpha$ ablation ${ }^{32}$.

Regulation of RXR expression and cellular localization. In view of their physiological importance, the mode of regulation of $R X R$ gene expression has quite paradoxically received little attention. Only the cloning and characterization of the promoter of the human $R X R A$ gene has been reported, revealing a TATA-less sequence with features of housekeeping genes ${ }^{33}$. Direct transcriptional regulation of $\mathrm{RXR} \alpha, \infty 0$ and $\propto$ gene expression is seen in severe sepsis and upon LPS 
or cytokine treatment in rodent heart and liver ${ }^{34}$. Interestingly, Rxra, Rxrb and Rxrg expression does not follow a rhythmic cycle of expression in metabolically active tissues from C57BI/6 mice, with the exception of Rxra in liver, and in opposition to a number of other RXR dimerization partners such as FXR, PPARs and RARs ${ }^{35}$. Finally, Rxrg1 expression is down-regulated in pituitary cells by 9-cis-RA through an atypical DR1 response element ${ }^{36}$.

In contrast, a number of reports documented the proteasomal degradation of $\mathrm{RXR} \alpha$ in various cellular backgrounds ${ }^{37}$, which appeared to be conditioned by RXR interaction with RAR and the corepressor SMRT ${ }^{38,39}$ and increased upon agonist binding ${ }^{40,41}$. Moreover, RXR $\alpha$ breakdown is dependent on MAPK or PKC activity ${ }^{42,43}$, hinting at a link between RXR $\alpha$ phosphorylation and proteasomal-mediated degradation ${ }^{44}$. The sensibility of $\operatorname{RXR} \alpha$ to proteasomal degradation is selective, since RXR $\beta$ is resistant to proteasome-mediated degradation in human embryonic kidney (HEK) cells ${ }^{42}$, in mouse 3T3-L1 preadipocytes and in visceral mouse and human adipose tissues ${ }^{45}$. $\mathrm{RXR} \alpha$, but not RXR $\beta$, is specifically conjugated to ubiquitin both in vitro and in vivo, leading to a marked, specific RXR $\alpha$ breakdown in obese visceral fat. Indeed, mouse and human obese visceral fat overexpress the ubiquitin hydrolase/ligase UCH-L1, which catalyzes, by a yet undefined molecular mechanism, $R X R \alpha$ ubiquitinylation and breakdown ${ }^{45}$. Quite similarly, $R X R \alpha$ is downregulated in many cancer cells and tissues and proteolytically degraded by cathepsin $L$ and/or calpain, generating a cytoplasmic, $\mathrm{N}$-terminally $44 \mathrm{kDa}$ truncated receptor able to interact with the protein kinase Akt and to activate the PI3K/Akt signalling pathway ${ }^{46}$. Interestingly, this $44 \mathrm{kDa}$ truncated RXR has also been located in the mitochondrial matrix in which it exerts a transcriptional activity ${ }^{47,48}$, suggesting that RXR can shuttle between the cytoplasmic and nuclear compartments. In line with this, RXR $\alpha$ possess a nuclear localization signal and associates to importin 80 , one of the cellular karyophilins binding the nuclear pore complex. When expressed as a GFP fusion protein, $R X R \alpha$ is mostly located in the nucleus but cytoplasmic RXR provides a piggyback transportation to the vitamin D receptor into the nucleus ${ }^{49,50}$. The nucleoplasmic sublocalization to the splicing factor compartment may also be another factor controlling RXR transcriptional activities ${ }^{51}$. LPS treatment of target cells can induce both downregulation of $R x r$ expression and its cytoplasmic relocalization ${ }^{52}$, providing a molecular basis for the observed repression of RXR target genes during TLR4-mediated inflammation. This cytoplasmic relocalization likely depends on $\mathrm{RXR} \alpha$ nuclear export signal located in its $\mathrm{DBD}$, which also 
conditions the RXR-mediated targeting of Nur77/NR4A1 and of the thyroid hormone receptor to mitochondria to promote apoptosis ${ }^{53,54}$. Thus, in addition to a simple, on-off regulatory mechanism of $\mathrm{RXR}$ expression, more subtle mechanisms affecting its cellular localization control RXR transcriptional activities and may generate novel functions for this multi-faceted nuclear receptor. Finally, fine-tuning of RXR transcriptional activity also relies on post-translational modifications (PTM): the MAPK-dependent phosphorylation of human $\mathrm{RXR} \alpha$ at serine 260 regulates coactivator recruitment to a RXR-VDR dimer ${ }^{55}$ and its degradation through the ubiquitin-proteasome pathway ${ }^{56}$, a PTM which can be controlled through the growth factor status of target cells ${ }^{57}$. In addition, RXR phosphorylation on distinct serine residues is dependant on its association with RAR and can affect the transcriptional cooperation between RAR and RXR \{Gianni, Tarrade, et al. 200318581 /id\}. This non exhaustive list of the role of PTMs on RXR activity suggest a great flexibility of RXR functions according to pathophysiological conditions which affect regulatory signaling pathways.

\section{RXR, a versatile dimerization partner.}

$\boldsymbol{R X R}$ dimers: RXRs establish dimeric complexes either spontaneously ${ }^{58}$ or in a ligand-dependent manner ${ }^{59}$ with a number of other NRs (Figure 2), resulting in distinct functional outcomes. While necessary for high affinity DNA binding of several NRs such as the T3R, vitamin D or RARs, FXR/RXR dimers display a strongly decreased DNA binding upon rexinoid ligation ${ }^{60}$. The DNA binding motifs to which RXR heterodimers bind are, in most cases, direct repeats (DR) containing the AGGTCA heptameric sequence and follow the so called 1 to 5 rule. According to this rule, RXR homodimers and PPAR-RXR heterodimers bind to DR1 motifs, whereas DR2 and DR5 are preferential binding motifs for RXR-RAR heterodimers, and DR3 and DR4 favor the association of vitamin D3 and T3 receptor heterodimers, respectively. This rule is however quite flexible since, for example, FXR-RXR dimers can also bind to inverted repeat (IR) motifs ${ }^{61}$ and RXR-RAR dimers to DR4 motifs ${ }^{62}$. It is however clear that the polarization of RXR heterodimers plays a crucial role in the transcriptional outcome upon ligand activation ${ }^{63}$.

RXR dimerization can also confer new biological properties: as mentioned above, RXR association with Nur77/NR4A1 promotes mitochondrial targeting of Nur77 and subsequent apoptosis

${ }^{64}$, whereas RXR association to the PML-RAR $\alpha$ fusion protein unleashes PML-RAR $\alpha$ oncogenic 
potential in hematopoietic cells ${ }^{65,66}$. Of note, a number of proteins involved in various biological processes have been shown to interact physically and/or functionnally with RXRs (Figure 2), paving the way for the discovery of novel RXR functions. The interaction of RXR with NPAS/MOP4 and Clock, two critical regulators of the circadian feedback loop ${ }^{67}$, suggest for example that circadian rhythms can be brought under the control of endogenous RXR ligands such as DHA, whose availability may depend on the nutritional status. As previously documented, NR-mediated transcription involves DNA double strand breakage and DNA repair ${ }^{68,69}$ and in this context, another role of RXR that could also be envisioned is the control of DNA repair through physical interaction with the $T: G$ mismatch-specific thymine-DNA glycosylase $\left(T D G,{ }^{70}\right.$ ), in analogy with the reported PCNA-RAR $\alpha$ interaction ${ }^{71}$.

RXRs' ability to homotetramerize or homodimerize has been proposed to be, at least in part, the molecular basis for anticarcinogenic or metabolic properties of rexinoids respectively ${ }^{2,3}$. The dissociation of RXR tetramers has been correlated to the ability of rexinoids to release DNA looping and to trigger apoptosis of breast cancer cells ${ }^{2}$. A PPAR $\alpha$-independent activation of DR1 response elements has been reported, suggesting that RXR $\alpha$ homodimers may substitute for PPAR $\alpha / R X R \alpha$ on PPAR response elements, and providing a plausible, but not formally proven, molecular basis for the activation of some PPAR $\alpha$ target genes by rexinoids in vivo ${ }^{3,72}$. Taken as a whole, these and other data show that RXRs actively contribute to the transcriptional activity of dimers in which they are incorporated, affecting multiple physiological and pathological processes.

Functional redundancy and the lack of specific RXR isotype-specific ligands hinder a detailed investigation of the specific functions, if any, of each RXR isotype. However, a few reports point to distinct functions for each isotype. We recently demonstrated that RXR $\alpha / P P A R \gamma$ heterodimers were much less sensitive to PPAR $\gamma$ agonism than $\operatorname{RXR} \beta / P P A R \gamma$ heterodimers, due to a strong, ligand-insensitive interaction of the SMRT corepressor with RXR $\alpha$-containing heterodimers ${ }^{45}$. RXR $\beta$ also displays peculiar heterodimerization properties in that it is unable to engage interactions with Nurr1, in contrast to $\mathrm{RXR} \alpha$ and $\mathrm{RXR} \gamma$, although the physiological relevance of such heterodimers has not been definitively established ${ }^{73}$. This call for developmental studies of dimerization-deficient Nurr 1 mice. Furthermore, the $\mathrm{RXR} \alpha$ and $\mathrm{RXR} \beta$ subtypes regulate differentially the transcriptional activity of T3R in a chimeric model ${ }^{74}$. In testis, RXR $\beta$ expression is confined to Sertoli cells, indicating a critical 
role in spermiation and cholesterol metabolism ${ }^{75,76}$. Rxrg1 ablation alters the response of cholinergic neurons from the nigrostriatal pathway ${ }^{77}$, in agreement with its expression pattern showing a preferential expression in the brain and skeletal muscle. RXR $\gamma$-deficient mice display high metabolic rates and resistance to weight gain when fed a high fat diet, which may stem either from an interference with the thyrotrope axis and/or skeletal muscle-specific effects ${ }^{78}$. These data show that RXR isotypes are involved in a plethora of biological responses which are tissue- and very likely isoform-specific. A more thorough investigation using conditional, tissue-specific KO mice would undoubtedly bring new insights into this complex RXR biology.

\section{RXR permissivity and metabolic regulations}

Although highly dependent on the model system used to assess this property, RXR-containing heterodimers can be categorized, quite schematically, as either "permissive" or "non-permissive", as they can be activated, or not, through the RXR moiety. Nuclear receptors with a high affinity for their cognate ligands are mostly found in the non-permissive category, such as RARs, T3R and VDR ${ }^{79}$. These receptors have a noticeable intrinsic repressive activity in the unliganded state. In contrast, low affinity-binding, lipid-activated nuclear receptors such as peroxisome proliferator-activated receptors (PPARs), the bile acid/farnesoid receptor (FXR) or oxysterol receptors (LXRs) are considered as permissive entities, suggesting that the functional control of RXR partners evolved to couple differentially endocrine and metabolic regulations ${ }^{80}$. The mechanism by which this molecular crosstalk takes place is still poorly defined, and is very certainly specific for each heterodimer: indeed, while the C-terminal activating function 2 ( $A F 2$, required for coactivator recruitment) of $R X R$ is dispensable for PPAR $\gamma$ synergistic activation, it is required for PPAR\&o and T3R activation ${ }^{81,82}$. The exact physical basis for this functional synergy is unknown, however, mutagenesis studies and structural studies suggest that one coactivator molecule is recruited per heterodimer, with a single LXXLL motif being anchored to each partner ${ }^{83}$. Intriguingly enough, the structural studies dismissed the possibility of allosteric phenomenons between heterodimeric partners, at least for RAR-RXR heterodimers ${ }^{84}$. In contrast, mutagenesis studies of the RXR-LXR complex spoke in favor of allosteric interactions between the two components of the dimer ${ }^{85}$, a difference which could explain the permissive nature of the LXR-RXR dimer. Importantly, the activation of a given permissive 
heterodimer through RXR does not equate activation through the RXR partner: the transcriptional response elicited through $\mathrm{RXR}$ overlaps only partially with that elicited through its partner ${ }^{86-88}$. In a detailed study, it was found that activation of LXR target genes in liver by a rexinoid was restricted to genes involved in lipogenesis, whereas those involved in cholesterol homeostasis were left unaffected. While providing a molecular explanation for the hypertriglyceridemic effect of bexarotene and possibly other rexinoids, these data raise the yet unresolved question of whether this reflects a selective permissivity of RXR-LXR heterodimer or another mechanism ${ }^{89}$. This may relate to the differential affinity of LXR and RXR for LXXLL-containing peptides ${ }^{90}$ and thus to the assembly of distinct coactivator complexes on each receptor monomer. Alternatively, selective post-translational modifications may control RXR permissivity in a promoter-specific context, in analogy to the protein kinase A-dependent desubordination of RXR in the RXR-RAR heterodimer ${ }^{16}$.

\section{Towards heterodimer-specific rexinoids}

RXRs can bind a variety of natural and synthetic derivatives, ranging from $\omega-3$ unsaturated fatty acids, the non steroidal anti-inflammatory drug sulindac to organotins ${ }^{46,91}$. A number of RXR agonists with no isotype selectivity have been synthesized, and much like for other NRs, the concept of selective nuclear receptor modulator (SNuRM) applies to this class of compounds. Indeed, the structural diversity of rexinoids translates into distinct patterns of gene expression induced upon treatment of target cells by these molecules ${ }^{92}$, as described previously for other $\mathrm{NRs}^{93}$. For example, the two rexinoids HX630 and LG268 induce only partially overlapping gene expression profiles when used in combination with a RAR agonist, Am580 ${ }^{88}$. Furthermore, LG268 is a rather promiscuous rexinoid, since able to activate both RXR-LXR and RXR-PPAR $\gamma$ dimers, whereas HX630 induces a preferential activation of RXR-PPAR $\gamma$ dimers ${ }^{94}$. Along the same line, the poorly active rexinoid $\mathrm{HX600}$ acts as an efficient activator of $\mathrm{RXR} \alpha-$ and $\mathrm{RXR} \gamma-\mathrm{Nur77/NR} 4 \mathrm{~A} 1$ dimers, but not of RXR $\beta$-Nur77 dimers, the latter feature being in line with the reported inability of $R X R \beta$ to dimerize with Nurr1/NR4A2 ${ }^{95}$. Interestingly, the rexinoid LG101506 activates selectively RXR-PPAR $\gamma$ heterodimers, but not RXR-RAR or RXR-LXR heterodimers ${ }^{15}$. In agreement with the predicted biological consequences of PPAR $\gamma$ activation, this compound lowered significantly blood glucose levels in genetically diabetic mice and did not increase blood triglyceride levels in Sprague-Dawley rats 
after a seven day treatment ${ }^{96}$. However, RXR antagonism in the RXR-PPAR $\gamma$ dimer has also proven to be efficient in normalizing glycemia and fat mass in animal models ${ }^{97}$, but this pharmacological option has not been explored further. Taken together, these data suggest that the design of heterodimer-specific rexinoids can be envisioned, although no precise rules have emerged yet for an efficient drug design.

\section{The RXR cistrome}

The recent availability of deep sequencing techniques led to the identification of RXR dimer DNA binding sites on a genome-wide scale. A consistent finding was that these potential regulatory sites are very often located at intronic locations at a distance from the transcriptional start site of regulated genes, calling for the re-examination of previous promoter studies which identified functional response elements in the vicinity of promoter regions. Another observation was that a vast majority of these heterodimer binding sites are prebound by RXR and that either ligand treatment or increased expression of the RXR partner lead to the expected heterodimer formation. This suggests a model in which RXR serves as a docking site for multiple nuclear receptors, and that the heterodimer composition will vary according to extra-or intracellular cues activating or repressing RXR and/or its dimerization partner. Furthermore, these data provide a general ground for the widely held assumption that RXR increases the DNA binding affinity of RXR-containing heterodimers ${ }^{98-100}$.

\section{Concluding remarks}

Heterodimerization is a general, simple mechanism by which a linear signaling pathway can be converted into a very complex network acting in a cell-specific manner in response to physiological or pathological cues. RXRs act as common heterodimerization partners for a number of other nuclear receptors, and as such are often considered as loosening the specificity of the signaling pathway. As a consequence, their usefulness as drug targets has been dismissed. However, the occurrence of several isotypes, and of multiple isoforms raise the possibility, as demonstrated by some reports described above, that RXR functions may be diverse and mediated through distinct molecular entities. An in-depth understanding of their respective biological functions, as well as the identification of 
heterodimer-selective synthetic compounds, will undoubtedly unravel novel aspect of RXR biology and pharmacology.

\section{Future directions box:}

- Is there specific, endogenous ligands for RXR?

- $\quad$ Can these ligands regulate specific metabolic pathways?

- What are the functions for each RXR isotype?

- $\quad$ Are RXRs only transcriptional regulators?

\section{Reference List}

1 Germain,P. et al. (2006) International Union of Pharmacology. LXIII. Retinoid X receptors. Pharmacol. Rev. 58, 760-772

2 Yasmin,R. et al. (2010) Inhibition of mammary carcinoma cell growth by RXR is mediated by the receptor's oligomeric switch. J. Mol. Biol. 397, 1121-1131

3 ljpenberg,A. et al. (2004) In vivo activation of PPAR target genes by RXR homodimers. EMBO J 23, 2083-2091

4 Dollé,P. (2009) Developmental expression of retinoic acid receptors (RARs). Nucl. Recept. Signal. 7, e006

5 Mascrez,B. et al. (2009) A transcriptionally silent RXRalpha supports early embryonic morphogenesis and heart development. Proc. Natl. Acad. Sci. U. S. A 106, 4272-4277

6 Kane,M.A. et al. (2005) Quantification of endogenous retinoic acid in limited biological samples by LC/MS/MS. Biochem. J. 388, 363-369

7 deUrquiza,A.M. et al. (2000) Docosahexaenoic acid, a ligand for the retinoid X receptor in mouse brain. Science 290, 2140-2144

8 Ziouzenkova,O. et al. (2007) Asymmetric cleavage of beta-carotene yields a transcriptional repressor of retinoid $\mathrm{X}$ receptor and peroxisome proliferator-activated receptor responses. Mol. Endocrinol. 21, 77-88 
9 Duvic,M. et al. (2001) Bexarotene is effective and safe for treatment of refractory advanced-stage cutaneous T-cell lymphoma: multinational phase II-III trial results. J. Clin. Oncol. 19, 2456-2471

$10 \mathrm{Vu}-\mathrm{Dac}, N$. et al. (1998) Retinoids increase human apo C-III expression at the transcriptional level via the retinoid $X$ receptor. Contribution to the hypertriglyceridemic action of retinoids. $J$ Clin. Invest $102,625-632$

11 Egea,P.F. et al. (2000) Crystal structure of the human RXR alpha ligand-binding domain bound to its natural ligand: 9-cis retinoic acid. EMBO J. 19, 2592-2601

12 Mukherjee,R. et al. (1997) Sensitization of diabetic and obese mice to insulin by retinoid X receptor agonists. Nature 386, 407-410

13 Cesario,R.M. et al. (2001) The rexinoid LG100754 is a novel RXR:PPARgamma agonist and decreases glucose levels in vivo. Mol. Endocrinol. 15, 1360-1369

14 Morita,K. et al. (2005) Selective allosteric ligand activation of the retinoid X receptor heterodimers of NGFI-B and Nurr1. Biochem. Pharmacol 71, 98-107

15 Leibowitz,M.D. et al. (2006) Biological characterization of a heterodimer-selective retinoid X receptor modulator: potential benefits for the treatment of type 2 diabetes. Endocrinology 147, 1044-1053

16 Altucci,L. et al. (2005) Rexinoid-triggered differentiation and tumor-selective apoptosis of acute myeloid leukemia by protein kinase A-mediated desubordination of retinoid $\mathrm{X}$ receptor. Cancer Res. $65,8754-8765$

17 Bookout,A.L. et al. (2006) Anatomical profiling of nuclear receptor expression reveals a hierarchical transcriptional network. Cell 126, 789-799

18 Brocard,J. et al. (1996) Two novel RXR alpha isoforms from mouse testis. Biochem. Biophys. Res. Commun. 229, 211-218

19 Fleischhauer,K. et al. (1992) Isolation of a full-length cDNA clone encoding a N-terminally variant form of the human retinoid X receptor beta. Nucleic Acids Res. 20, 1801

20 Fujita,A. and Mitsuhashi,T. (1999) Differential regulation of ligand-dependent and ligand-independent functions of the mouse retinoid $\mathrm{X}$ receptor beta by alternative splicing. Biochem. Biophys. Res. Commun. 255, 625-630

\footnotetext{
${ }^{5}$ Present address: Centre des Neurosciences Pariis2Sud, CNRS UMR 1197, Université Paris Sud-XI, F-91405 ORSAY, France
} 
21 Liu,Q. and Linney,E. (1993) The mouse retinoid-X receptor-gamma gene - genomic organization and evidence for functional isoforms. Mol. Endocrinol. 7, 651-658

22 Mark,M. et al. (2006) Function of retinoid nuclear receptors: lessons from genetic and pharmacological dissections of the retinoic acid signaling pathway during mouse embryogenesis. Annu. Rev. Pharmacol. Toxicol. 46, 451-480

23 Krezel,W. et al. (1996) RXR gamma null mice are apparently normal and compound RXR alpha +/-/RXR beta -/-/RXR gamma -/- mutant mice are viable. Proc. Natl Acad. Sci. U. S. A 93, 9010-9014

24 Wan,Y.J. et al. (2003) Hepatocyte retinoid X receptor-alpha-deficient mice have reduced food intake, increased body weight, and improved glucose tolerance. Endocrinology 144, 605-611

25 Cai,Y. et al. (2002) The role of hepatocyte RXR alpha in xenobiotic-sensing nuclear receptor-mediated pathways. Eur. J Pharm. Sci. 15, 89-96

26 Wan,Y.J. et al. (2000) Hepatocyte-specific mutation establishes retinoid X receptor alpha as a heterodimeric integrator of multiple physiological processes in the liver. Mol. Cell Biol. 20, 4436-4444 27 Yang,X. et al. (2010) Deregulation of growth factor, circadian clock, and cell cycle signaling in regenerating hepatocyte RXRalpha-deficient mouse livers. Am. J. Pathol. 176, 733-743

28 Imai,T. et al. (2001) Selective ablation of retinoid X receptor alpha in hepatocytes impairs their lifespan and regenerative capacity. Proc. Natl. Acad. Sci. U. S. A. 98, 4581-4586

$29 \mathrm{Li}, \mathrm{M}$. et al. (2001) RXR-alpha ablation in skin keratinocytes results in alopecia and epidermal alterations. Development 128, 675-688

30 Ocadiz-Delgado,R. et al. (2008) Impaired cervical homeostasis upon selective ablation of RXRalpha in epithelial cells. Genesis. 46, 19-28

31 Ricote,M. et al. (2006) Normal hematopoiesis after conditional targeting of RXRalpha in murine hematopoietic stem/progenitor cells. J. Leukoc. Biol. 80, 850-861

32 Metzger,D. et al. (2005) Functional role of RXRs and PPARgamma in mature adipocytes. Prostaglandins Leukot. Essent. Fatty Acids 73, 51-58

$33 \mathrm{Li}, \mathrm{G}$. et al. (2000) Cloning and characterization of the human retinoid $\mathrm{X}$ receptor alpha gene: Conservation of structure with the mouse homolog. Biochem. Biophys. Res. Commun. 269, 54-57 34 Feingold,K. et al. (2004) Altered expression of nuclear hormone receptors and coactivators in mouse heart during the acute-phase response. Am. J. Physiol Endocrinol. Metab 286, E201-E207 
35 Yang,X. et al. (2006) Nuclear receptor expression links the circadian clock to metabolism. Cell 126, $801-810$

36 McDermott,N.B. et al. (2002) Isolation and functional analysis of the mouse RXRgamma1 gene promoter in anterior pituitary cells. J. Biol. Chem. 277, 36839-36844

37 Prufer,K. and Barsony,J. (2002) Retinoid X Receptor Dominates the Nuclear Import and Export of the Unliganded Vitamin D Receptor. Mol Endocrinol 16, 1738-1751

38 Gianni,M. et al. (2003) The AF-1 and AF-2 Domains of RAR\{gamma 2 and RXR\{alpha $\}$ Cooperate for Triggering the Transactivation and the Degradation of RAR\{gamma\}2/RXR\{alpha\} Heterodimers. J. Biol. Chem. 278, 34458-34466

39 Boudjelal,M. et al. (2000) Ubiquitin/proteasome pathway regulates levels of retinoic acid receptor gamma and retinoid X receptor alpha in human keratinocytes. Cancer Res. 60, 2247-2252

40 Pettersson,F. et al. (2008) Rexinoids modulate SXR activity by increasing its protein turnover in a calpain-dependent manner. J. Biol. Chem. 283, 21945-21952

$41 \mathrm{Lin}, \mathrm{J} . \mathrm{H}$. et al. (2008) Clinical and in vitro resistance to bexarotene in adult T-cell leukemia: loss of RXR-alpha receptor. Blood 112, 2484-2488

42 Tsao,W.C. et al. (2005) Proteasome inhibitors induce peroxisome proliferator-activated receptor transactivation through RXR accumulation and a protein kinase C-dependent pathway. Exp. Cell Res. $304,234-243$

43 Lattuada,D. et al. (2007) Accumulation of retinoid X receptor-alpha in uterine leiomyomas is associated with a delayed ligand-dependent proteasome-mediated degradation and an alteration of its transcriptional activity. Mol. Endocrinol. 21, 602-612

44 Matsushima-Nishiwaki,R. et al. (2001) Phosphorylation of retinoid X receptor alpha at serine 260 impairs its metabolism and function in human hepatocellular carcinoma. Cancer Res. 61, 7675-7682 45 Lefebvre,B. et al. (2010) Proteasomal degradation of retinoid X receptor alpha reprograms transcriptional activity of PPARgamma in obese mice and humans. J. Clin. Invest 120, 1454-1468.

46 Zhou,H. et al. (2010) NSAID sulindac and its analog bind RXRalpha and inhibit RXRalpha-dependent AKT signaling. Cancer Cell 17, 560-573

47 Casas,F. et al. (2003) Endocrine regulation of mitochondrial activity: involvement of truncated RXRalpha and c-Erb Aalpha1 proteins. FASEB J. 17, 426-436 
48 Lin,Y.W. et al. (2008) 9-cis retinoic acid induces retinoid $X$ receptor localized to the mitochondria for mediation of mitochondrial transcription. Biochem. Biophys. Res. Commun. 377, 351-354

49 Yasmin, R. et al. (2005) Nuclear import of the retinoid X receptor, the vitamin D receptor, and their mutual heterodimer. J. Biol. Chem. 280, 40152-40160

50 Prufer,K. et al. (2000) Dimerization with retinoid X receptors promotes nuclear localization and subnuclear targeting of vitamin D receptors. J. Biol. Chem. 275, 41114-41123

51 Tanaka,T. et al. (2004) Altered Localization of Retinoid X Receptor \{alpha\} Coincides with Loss of Retinoid Responsiveness in Human Breast Cancer MDA-MB-231 Cells. Mol. Cell. Biol. 24, 3972-3982 52 Ghose,R. et al. (2004) Endotoxin leads to rapid subcellular re-localization of hepatic RXRalpha: A novel mechanism for reduced hepatic gene expression in inflammation. Nucl. Recept. 2, 4

53 Cao,X. et al. (2004) Retinoid X receptor regulates Nur77/TR3-dependent apoptosis by modulating its nuclear export and mitochondrial targeting. Mol Cell Biol 24, 9705-9725

54 Lin,X.F. et al. (2004) RXRalpha acts as a carrier for TR3 nuclear export in a 9-cis retinoic acid-dependent manner in gastric cancer cells. J. Cell Sci. 117, 5609-5621

55 Macoritto,M. et al. (2008) Phosphorylation of the human retinoid X receptor alpha at serine 260 impairs coactivator(s) recruitment and induces hormone resistance to multiple ligands. J. Biol. Chem. $283,4943-4956$

56 Adachi,S. et al. (2002) Phosphorylation of retinoid X receptor suppresses its ubiquitination in human hepatocellular carcinoma. Hepatology 35, 332-340

57 Shankaranarayanan,P. et al. (2009) Growth factor-antagonized rexinoid apoptosis involves permissive PPARgamma/RXR heterodimers to activate the intrinsic death pathway by NO. Cancer Cell 16, 220-231

58 Feige,J.N. et al. (2005) Fluorescence imaging reveals the nuclear behavior of peroxisome proliferator-activated receptor/retinoid X receptor heterodimers in the absence and presence of ligand. J. Biol. Chem. 280, 17880-17890

59 Depoix,C. et al. (2001) Control of retinoic acid receptor heterodimerization by ligand-induced structural transitions. a novel mechanism of action for retinoid antagonists. J. Biol. Chem. 276, $9452-9459$ 
60 Kassam,A. et al. (2003) Retinoid X Receptor (RXR) Agonist-induced Antagonism of Farnesoid X Receptor (FXR) Activity due to Absence of Coactivator Recruitment and Decreased DNA Binding. J. Biol. Chem. 278, 10028-10032

61 Laffitte,B.A. et al. (2000) Identification of the DNA binding specificity and potential target genes for the farnesoid X-activated receptor. J. Biol. Chem. 275, 10638-10647

62 Sanguedolce,M.V. et al. (1997) The promoter context is a decisive factor in establishing selective responsiveness to nuclear class II receptors. EMBO J. 16, 2861-2873

63 Kurokawa,R. et al. (1995) Polarity-specific activities of retinoic acid receptors determined by a co-repressor. Nature 377, 451-454

64 Zhang,X.K. (2007) Targeting Nur77 translocation. Expert. Opin. Ther. Targets. 11, 69-79

65 Zhu,J. et al. (2007) RXR is an essential component of the oncogenic PML/RARA complex in vivo. Cancer Cell 12, 23-35

66 Zeisig,B.B. et al. (2007) Recruitment of RXR by homotetrameric RARalpha fusion proteins is essential for transformation. Cancer Cell 12, 36-51

67 McNamara,P. et al. (2001) Regulation of CLOCK and MOP4 by nuclear hormone receptors in the vasculature: a humoral mechanism to reset a peripheral clock. Cell 105, 877-889

68 Kress,C. et al. (2006) Active cytosine demethylation triggered by a nuclear receptor involves DNA strand breaks. Proc. Natl. Acad. Sci. U. S. A 103, 11112-11117

69 Ju,B.G. et al. (2006) A topoisomerase Ilbeta-mediated dsDNA break required for regulated transcription. Science 312, 1798-1802

70 Um,S. et al. (1998) Retinoic acid receptors interact physically and functionally with the T:G mismatch-specific thymine-DNA glycosylase. J. Biol. Chem. 273, 20728-20736

71 Martin,P.J. et al. (2005) The proliferating cell nuclear antigen regulates retinoic acid receptor transcriptional activity through direct protein-protein interaction. Nucl. Acids. Res. 33, 4311-4321

72 Takahashi,Y. et al. (2009) ApoC-III gene expression is sharply increased during adipogenesis and is augmented by retinoid $X$ receptor (RXR) agonists. FEBS Lett. 583, 493-497

73 Sacchetti,P. et al. (2002) Requirements for heterodimerization between the orphan nuclear receptor Nurr1 and retinoid X receptors. J. Biol. Chem. 277, 35088-35096 
$74 \mathrm{Li}, \mathrm{D}$. et al. (2004) Novel roles of retinoid $X$ receptor $(R X R)$ and $R X R$ ligand in dynamically modulating the activity of the thyroid hormone receptor/RXR heterodimer. J. Biol. Chem. 279, 7427-7437

75 Vernet,N. et al. (2006) Retinoic acid metabolism and signaling pathways in the adult and developing mouse testis. Endocrinology 147, 96-110

76 Mascrez,B. et al. (2004) Ligand-dependent contribution of RXRbeta to cholesterol homeostasis in Sertoli cells. EMBO Rep. 5, 285-290

77 Saga,Y. et al. (1999) Impaired extrapyramidal function caused by the targeted disruption of retinoid X receptor RXRgamma1 isoform. Genes Cells 4, 219-228

78 Haugen,B.R. et al. (2004) Retinoid X receptor gamma-deficient mice have increased skeletal muscle lipoprotein lipase activity and less weight gain when fed a high-fat diet. Endocrinology 145, 3679-3685

79 Shulman,A.I. and Mangelsdorf,D.J. (2005) Retinoid X receptor heterodimers in the metabolic syndrome. N. Engl. J. Med. 353, 604-615

80 Shulman,A.I. et al. (2004) Structural determinants of allosteric ligand activation in RXR heterodimers. Cell 116, 417-429

81 Castillo,A.I. et al. (2004) A permissive retinoid X receptor/thyroid hormone receptor heterodimer allows stimulation of prolactin gene transcription by thyroid hormone and 9-cis-retinoic acid. Mol. Cell. Biol. 24, 502-513

82 Calleja,C. et al. (2006) Genetic and pharmacological evidence that a retinoic acid cannot be the RXR-activating ligand in mouse epidermis keratinocytes. Genes Dev. 20, 1525-1538

83 Leo,C. et al. (2001) Role of retinoid receptor coactivator pockets in cofactor recruitment and transcriptional regulation. J. Biol. Chem. 276, 23127-23134

84 Pogenberg,V. et al. (2005) Characterization of the Interaction between Retinoic Acid Receptor/Retinoid X Receptor (RAR/RXR) Heterodimers and Transcriptional Coactivators through Structural and Fluorescence Anisotropy Studies. J. Biol. Chem. 280, 1625-1633

85 Son,Y.L. and Lee,Y.C. (2009) Molecular determinants of the interactions between LXR/RXR heterodimers and TRAP220. Biochem. Biophys. Res. Commun. 384, 389-393 
86 Anderson,S.P. et al. (2004) Overlapping Transcriptional Programs Regulated by the Nuclear Receptors Peroxisome Proliferator-Activated Receptor \{alpha\}, Retinoid X Receptor, and Liver X Receptor in Mouse Liver. Mol Pharmacol 66, 1440-1452

87 Zhang-Gandhi,C.X. and Drew,P.D. (2007) Liver X receptor and retinoid X receptor agonists inhibit inflammatory responses of microglia and astrocytes. J. Neuroimmunol. 183, 50-59

88 Ishida,S. et al. (2003) Clinically potential subclasses of retinoid synergists revealed by gene expression profiling. Mol. Cancer Ther. 2, 49-58

89 Lalloyer,F. et al. (2009) Rexinoid bexarotene modulates triglyceride but not cholesterol metabolism via gene-specific permissivity of the RXR/LXR heterodimer in the liver. Arterioscler. Thromb. Vasc. Biol. 29, 1488-1495

90 Son,Y.L. et al. (2008) RXR heterodimerization allosterically activates LXR binding to the second NR box of activating signal co-integrator-2. Biochem. J. 410, 319-330

91 Lemaire,M.A. et al. (2009) Activation of RXR-PPAR heterodimers by organotin environmental endocrine disruptors. EMBO Rep. 10, 367-373

92 Kagechika,H. and Shudo,K. (2005) Synthetic retinoids: recent developments concerning structure and clinical utility. J. Med. Chem. 48, 5875-5883

93 Gervois,P. et al. (2007) Drug Insight: mechanisms of action and therapeutic applications for agonists of peroxisome proliferator-activated receptors. Nat. Clin. Pract. Endocrinol Metab 3, 145-156 94 Nishimaki-Mogami,T. et al. (2008) The RXR agonists PA024 and HX630 have different abilities to activate LXR/RXR and to induce ABCA1 expression in macrophage cell lines. Biochem. Pharmacol. $76,1006-1013$

95 Sacchetti,P. et al. (2006) Multiple signaling pathways regulate the transcriptional activity of the orphan nuclear receptor NURR1. Nucl. Acids. Res. 34, 5515-5527

96 Michellys,P.Y. $\quad$ et al. (2003) Novel (2E,4E,6Z)-7-(2-alkoxy-3,5-dialkylbenzene)-3-methylocta-2,4,6-trienoic acid retinoid $X$ receptor modulators are active in models of type 2 diabetes. J. Med. Chem. 46, 2683-2696

97 Yamauchi,T. et al. (2001) Inhibition of RXR and PPARgamma ameliorates diet-induced obesity and type 2 diabetes. J. Clin. Invest 108, 1001-1013 
98 Meyer,M.B. et al. (2010) Genome-wide analysis of the VDR/RXR cistrome in osteoblast cells provides new mechanistic insight into the actions of the vitamin D hormone. J. Steroid Biochem. Mol. Biol. Feb. 18 (Epub ahead of print)

99 Nielsen,R. et al. (2008) Genome-wide profiling of PPARgamma:RXR and RNA polymerase II occupancy reveals temporal activation of distinct metabolic pathways and changes in RXR dimer composition during adipogenesis. Genes Dev. 22, 2953-2967

100 Hamza,M.S. et al. (2009) De-novo identification of PPARgamma/RXR binding sites and direct targets during adipogenesis. PLoS. One. 4, e4907

\section{Acknowledgements:}

Work in our laboratory is supported by grants from the European Union (Xtranet), Région Nord-Pas de Calais, FEDER, and Fondation Coeur et Artères. 


\section{FIGURE LEGENDS}

Figure 1: RXR sequences and structures. A) RXR primary structure. RXR isotypes have a structural organization similar to that of other nuclear receptors but lack a C-terminal F domain. AF-1: Activation Function-1; DBD: DNA Binding Domain; LBD: Ligand Binding Domain; AF-2: Activating Function 2; AF2-AD: AF2 activation domain (corresponding to helix 12 of the LBD). Splice variant names are indicated on the left, while the structure of the encoded protein is indicated on the right. B) Tertiary structure of the unliganded (Apo-RXR $\alpha$ ) and of the liganded RXR $\alpha$ LD to docosahexanoic acid (Holo-RXR $\alpha$ ) complexed to a SRC2 peptide. Coordinates were taken from the RCSB protein databank (1LBD and 1MV9) and visualized using the JMol software. C) Quaternary structure of a full length PPAR $\gamma-R X R \alpha$ heterodimer. Coordinates (3DZY) and molecule structure were obtained as described in B).

Figure 2: The RXR $\alpha$ interaction network. Proteins engaging physical and functional interactions with $\mathrm{RXR} \alpha$ are indicated and classified according to their main functions. Data were compiled from the visANT database [Hu, Z. et al., 2007, Towards zoomable multidimensional maps of the cell. Nat Biotechnol, 25(5): 547-54] and a PubMed search. 
A) Splice variant (Ensembl)

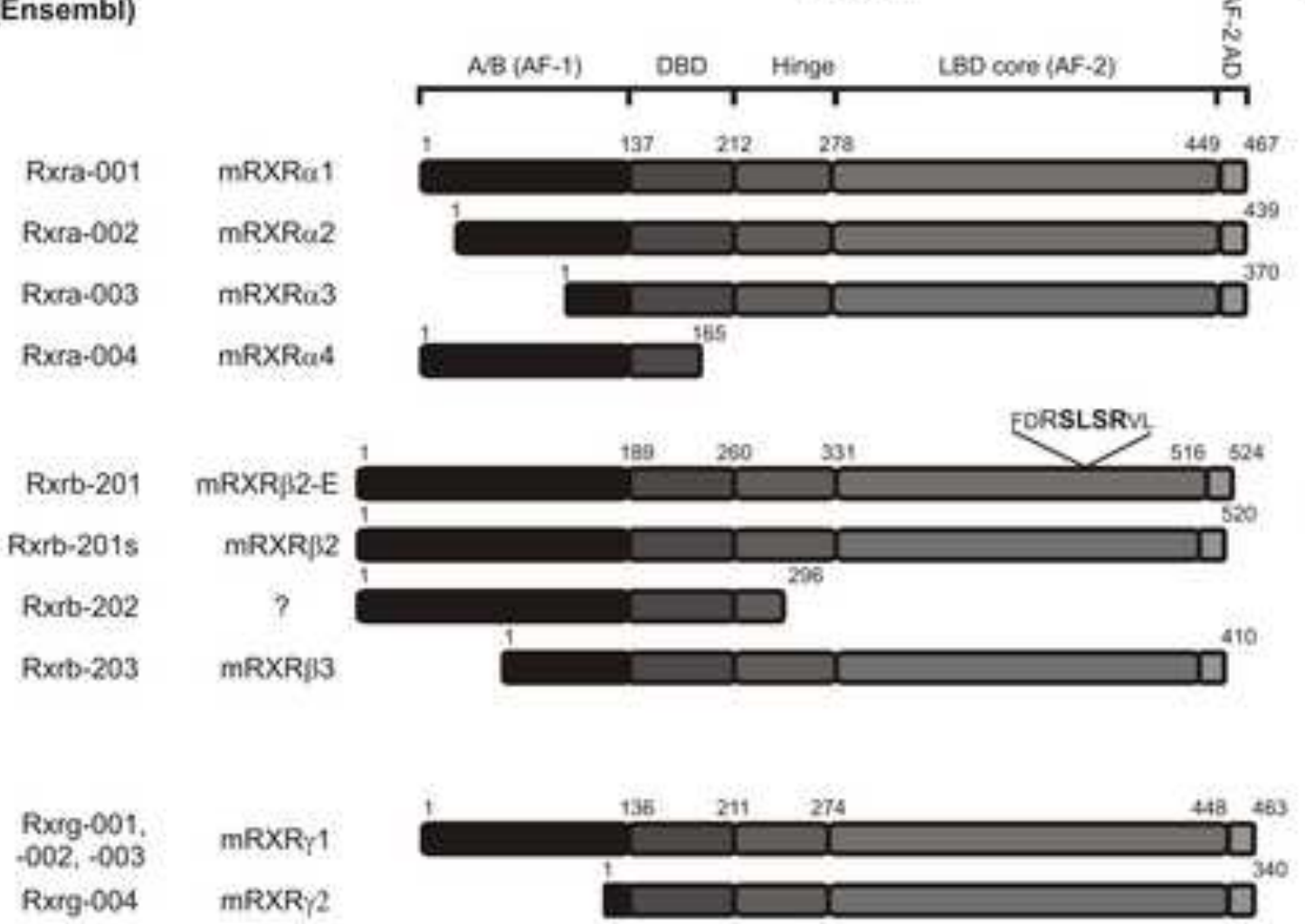

B)

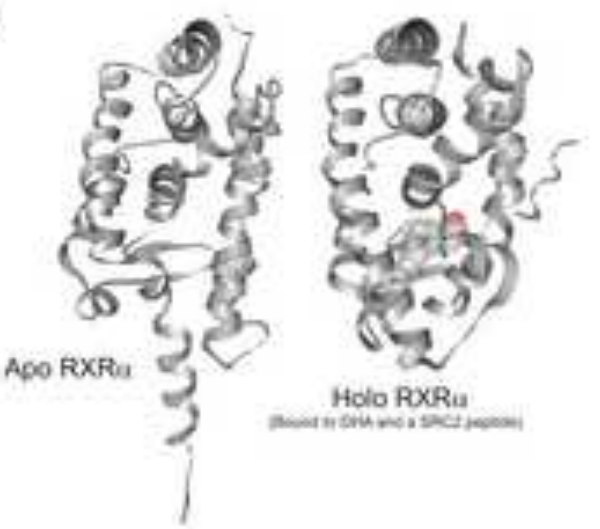

C)

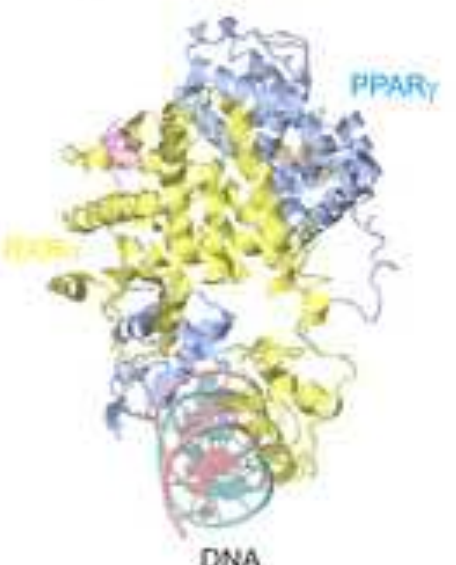

Lefebvre et al., Figure 1 


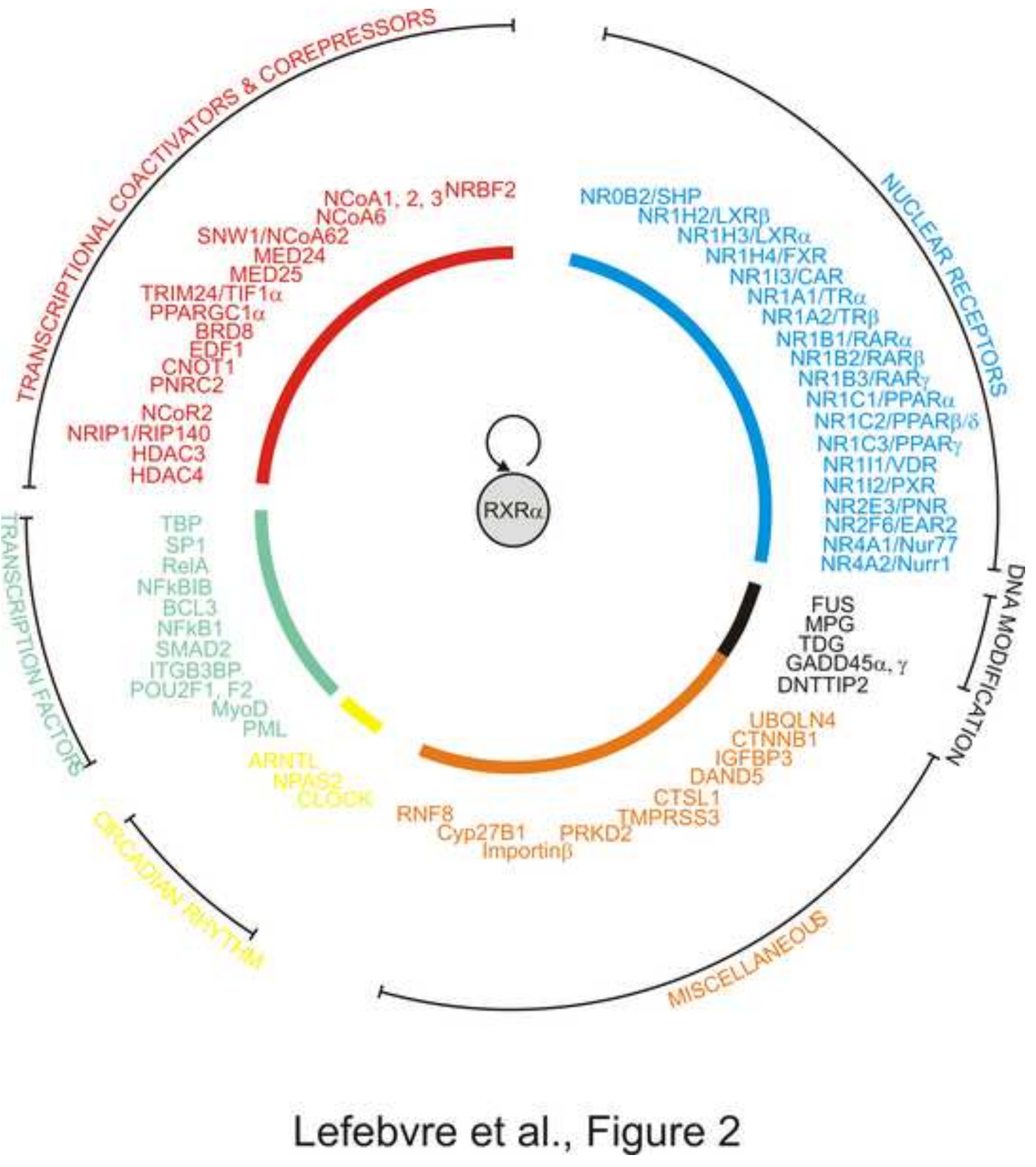

